\title{
The epidemiology of heart failure in the general Australian community - study of heart failure in the Australian primary carE setting (SHAPE): methods
}

Richard Whaddon Parsons', Danny Liew², A. Munro Neville ${ }^{1 *}$ (D, Ralph G. Audehm³ , Deepak Haikerwal ${ }^{4}$, Peter Piazza ${ }^{5}$, Kevin Lim ${ }^{6}$ and Andrew P. Sindone ${ }^{7}$

\begin{abstract}
Background: There is a paucity of information on the epidemiology of heart failure (HF) in Australia. The Study of Heart failure in the Australian Primary carE setting (SHAPE) study aims to estimate the prevalence and annual incidence of HF in the general Australian community and to describe the demographic and key clinical profile of Australians with HF.
\end{abstract}

Methods: We undertook a retrospective cohort study based on analysis of non-identifiable medical records of adult patients cared for at 43 general practices between 1 July 2013 and 30 June 2018. Data were extracted from coded (diagnosis, pathology and prescription fields) and uncoded fields (clinical notes) in the medical records. The latter searches of free text looked for common synonyms relevant to HF. The population was stratified into three groups based on a hierarchy of selection criteria: (1) definite HF, (2) probable HF and (3) possible HF. The prevalence and annual incidence of HF were calculated, along with 95\% confidence intervals.

Results: The practices provided care to 2.3 million individual patients over the five-year study period, of whom 1.93 million were adults and 1.12 million were regular patients. Of these patients 15,468 were classified as having 'definite HF', 4751 as having 'probable HF' and 33,556 as having 'possible HF'. A further 39,247 were identified as having an aetiological condition associated with HF.

A formal HF diagnosis, HF terms recorded as text in the notes and HF-specific medication were the most common methods to identify 'definite' HF patients. Typical signs and symptoms in combination with a diuretic prescription was the most common method to identify 'probable HF' patients. The majority of 'possible' HF patients were identified by the presence of 2 or more of the typical signs or symptoms. Dyspnoea was the commonest recorded symptom and an elevated jugular venous pressure the commonest recorded sign.

(Continued on next page)

\footnotetext{
* Correspondence: munro.neville@austrials.com.au

${ }^{1}$ AusTrials, Brisbane, Australia

Full list of author information is available at the end of the article
} 
(Continued from previous page)

Conclusions: This novel approach to undertaking retrospective research of primary care data successfully analysed a combination of coded and uncoded data from the electronic medical records of patients routinely managed in the GP setting. SHAPE is the first real-world study of the epidemiology of HF in the general Australian community setting.

Keywords: Retrospective, Heart failure, HF, Primary care, Australia, Epidemiology, Prevalence, Incidence, Demographics, SHAPE

\section{Background}

Currently, heart failure (HF) is estimated to affect 480, 000 Australians, with over 60,000 new diagnoses made every year [1]. The continued ageing of the Australian population is expected to further increase the burden of HF on the healthcare system in general and on primary care more specifically [2]. Of patients hospitalised with HF, only $20 \%$ are subsequently enrolled in hospitalbased disease management programs, with access especially limited in regional and remote communities [3]. The remaining $80 \%$ of patients are mostly reliant upon their general practitioners (GPs) for ongoing management of their HF.

In Australia, GPs are the healthcare providers who coordinate and monitor the care of patients with HF, including for follow up and referral to specialist care, such as cardiologists and renal physicians [4]. To date, little information has been gathered regarding the prevalence and incidence of HF in the general Australian community, as well as the demographic and clinical profiles of patients with HF. Such information is important for healthcare planning, as well as for establishing a baseline against which to compare future epidemiological data. Furthermore, insight is needed into areas in which the management of HF can be improved. There are data suggesting that many patients with HF are not optimally treated in primary care, with the majority of patients not being on the most appropriate therapies or at target doses $[5,6]$.

The Study of Heart failure in the Australian Primary carE setting (SHAPE) is a retrospective cohort study of primary care data that seeks to estimate the prevalence and annual incidence of HF in the Australian general community and to describe the demographic and key clinical profile of Australians with prevalent HF. The present article describes the methods undertaken in SHAPE and provides an overview of the main epidemiological findings.

\section{Methods}

We undertook a retrospective cohort study based on analysis of existing medical records of adult patients cared for at participating general practices between 1 July 2013 and 30 June 2018. All practices provided fully subsidised care to their patients (which is known as 'bulk-billing'). Participating practices were those within the Healius network (previously known as Primary Health Care) that used Medical Director software - this group comprised 43 centres from a network of 71 . The remaining 28 practices were using software other than Medical Director and so data were not available for extraction and analysis. All centres in the network are transitioning over to the Medical Director software according to a schedule. There were no other regional or socioeconomic differences between those included in our study and those omitted for software reasons.

The use of prescribing and electronic health records in Australian general practices has been widely adopted such that by 2005, Australian general practice had achieved near-universal clinical computerisation $[7,8]$. Medical Director software is one of two dominant providers of practice software in Australia, providing 4300 GP practices and 13,600 GP users with its practice software [9].

To identify patients with HF, a search of records was undertaken using Structured Query Language (SQL). The list of screening words was broad so that cases would be unlikely be missed. Search terms to identify a cohort for extraction and full analysis included HF diagnostic terms, HF-specific medication use, signs and symptoms of $\mathrm{HF}$, pathology test results indicative of $\mathrm{HF}$, the diagnosis of an aetiological condition for HF (Table 1) and a referral for cardiac imaging, principally echocardiography. These criteria were developed with expert opinion advice or from current Australian HF evidence-based guidelines [10]. All patients visiting the practices (with and without heart failure) were included and heart failure hospitalisation was not a prerequisite for being included in the study.

The cases were de-identified, removing all potentially identifiable data from the records, then provided to the researchers for analysis. Data were extracted from the following fields in the medical records: diagnosis, reason for presentation, prescriptions, vital signs, pathology results, specialist referrals and clinical notes. Chronic disease management item numbers billed to Medicare were also extracted. Each patient was allocated a unique study number so that re-identification would be possible by 
Table 1 Comorbidities that are an aetiological condition for HF

\begin{tabular}{ll}
\hline Coronary artery disease & Lysosomal storage disease \\
Coronary heart disease & Fabry disease \\
Peripheral vascular disease & Thyroid insufficiency \\
Peripheral arterial disease & Hyperactive thyroid \\
Myocardial infarction & Thyrotoxicosis \\
Unstable angina & Hashimoto's thyroiditis \\
Acute coronary syndrome & Grave's disease \\
Cardiac dysrhythmia / cardiac arrhythmia & Cushing's syndrome \\
Atrial fibrillation and atrial flutter & Adrenal insufficiency \\
Ischaemic heart disease & Diabetes mellitus \\
Myocardial ischaemia & Pheochromocytoma \\
Microvascular disease & Malnutrition \\
Myocyte stunning & Hypertension \\
Myocyte hibernation & Pericarditis \\
Myocarditis & Pericardial constriction \\
Cardiomyopathy & Pericardial effusion \\
Heart valve disease & Hypereosinophilic syndromes \\
Alcoholism & Endomyocardial fibrosis \\
Cardiac malignancy & Fibroelastosis \\
Arteriovenous fistula & Anaemia \\
Amyloidosis & Sepsis \\
Haemochidosis & Paget disease \\
Glycogen overload & Vitamin B deficiency \\
\hline Source: SHAPE & Promic fluid overload \\
\hline
\end{tabular}

Source: SHAPE Project Collaborators Expert Opinion, September 2018

Healius for future scrutiny of records for any reason (for example, missing data). This allowed records belonging to the same patient to be linked through time so that GP visits and management for each patient could be identified.

To ensure data integrity, consistency and completeness of the data extraction, a detailed quality control process was performed. A registered nurse who was an experienced study coordinator employed by Healius examined the records of a random sample of 50 identified patients to ensure that the query collected the correct data from the correct patients. The study coordinator also performed a disease register search of HF to make sure that the query did not omit from the extract any potential HF patients. This quality control process confirmed that the data extraction produced the correct patient level results and showed that the query was comprehensive so that HF patients were very unlikely to be omitted.

The study's primary endpoints were the prevalence and incidence of HF, stratified by age and gender, and standardised to the 2017 Australian population. We also sought to determine the demographics of the HF population and their clinical characteristics, including aetiological factors, comorbidities, symptoms of HF, examination findings and medication use. Other factors examined included the proportion receiving HF medications, the proportion receiving medications that are contraindicated in HF, the frequency of GP visits, the use of GP chronic disease management Medicare services, the use of mental health services, and the frequency of referrals from GPs to specific types of specialists. In the primary analyses, data comprised only 'active' patients; that is those patients who visited the medical centres at least three times over a two-year period [11].

Included patients were those who were aged 18 years and above, and who had one or more of the following criteria recorded in their medical record: i) a specific diagnosis of HF (Table 2); ii) were receiving ongoing treatment with a HF-specific medication (Table 3); iii) presented with signs or symptoms of HF (Table 4 and Table 5); or iv) had pathology test results indicative of HF (Table 6 and Table 7). In Australia, the HF-specific medications listed in Table 3 have a 'Restricted Benefit' in the Pharmaceutical Benefits Scheme (Australia's list of subsidised medications) to 'moderate to severe heart failure'. Furthermore, the restriction stipulates that patients must be stabilised on conventional therapy, which must include an angiotensin converting enzyme inhibitor or angiotensin II antagonist, if tolerated [12]. In the search of text fields, certain criteria were selected for common synonyms, which are listed in Additional file 1 Appendix - Free text search terms. If certain words preceded the selected words in the notes, then the

Table 2 Heart failure diagnosis terms

Past History Diagnosis or Reason for Presentation Keywords for Inclusion

Heart failure Systolic heart failure

Chronic cardiac Systolic dysfunction

failure

Chronic heart Diastolic heart failure

failure

Congestive Diastolic dysfunction

cardiac failure

CCF

Congestive heart HFrEF / Heart failure with reduced ejection fraction failure

$\mathrm{CHF}$

Cardiac failure HFpEF / Heart failure with preserved ejection fraction

Chronic heart Pulmonary oedema

failure

Left ventricular Ischaemic cardiomyopathy

failure

Right ventricular Dilated cardiomyopathy (but excluding hypertrophic failure cardiomyopathy)

Source: SHAPE Project Collaborators Expert Opinion, September 2018 
Table 3 Heart failure specific medications

\begin{tabular}{l} 
Ivabradine \\
Ethacrynic acid \\
Eplerenone \\
Bisoprolol \\
Nebivolol \\
Carvedilol \\
Metoprolol succinate (HF doses only $)$ \\
Sacubitril / Valsartan \\
\hline
\end{tabular}

Source: SHAPE Project Collaborators Expert Opinion, September 2018 In Australia, these medications have a restricted use benefit in the Pharmaceutical Benefits Scheme to 'moderate to severe heart failure' only. For example: https://www.pbs.gov.au/medicine/item/8733P for metoprolol succinate

${ }^{a}$ Doses $23.75 \mathrm{mg}, 47.5 \mathrm{mg}, 95 \mathrm{mg}, 190 \mathrm{mg}$ (controlled release)

condition was considered not to be present in those notes. For example, if there was a mention of 'shortness of breath (SOB)', but this was preceded by 'No', 'Nil', or 'denies', then SOB was considered not to be a problem for the patient at that time.

The search term 'PND' was found to produce a lot of false positive results (also being used for other conditions, such as post-natal depression, and post-nasal drip). A review of 2000 records with 'PND' was undertaken and this included 1151 with nasal symptoms, 659 with upper respiratory tract infection (URTI), 515 with sinusitis, 169 with lower respiratory tract infection (LRTI) and 63 with depression. However, the term 'PND' was still included, but non-HF causes were excluded and further supporting evidence (ejection fraction data, BNP data, or loop diuretic use) was required in order for a case to be classified as definite or probable HF.

The analysis assessed the number and combinations of relevant terms and cut-off criteria in a hierarchical approach. The population was then stratified into three groups based on a hierarchy of selection criteria: (1) definite HF, (2) probable HF and (3) possible HF (Table 8). The eligibility criteria for 'definite HF' were: HF coded in the field of diagnosis codes; any mention of HF diagnoses in the free text fields; prescription of HF-specific

Table 4 Typical and specific symptoms and signs of heart failure

\begin{tabular}{ll}
\hline Symptoms of heart failure & Signs of heart failure \\
\hline Dyspnoea (usually with exertion) & Elevated jugular venous pressure \\
Orthopnoea & Hepatojugular reflux \\
Paroxysmal nocturnal dyspnoea & Third heart sound \\
& Laterally displaced apex beat
\end{tabular}

Source: Atherton J, Sindone A, De Pasquale C, et al. National Heart Foundation of Australia and Cardiac Society of Australia and New Zealand Guidelines for the prevention, detection and management of heart failure in Australia 2018
Table $\mathbf{5}$ Less typical symptoms and signs of heart failure

\begin{tabular}{ll}
\hline Symptoms & Signs \\
\hline Nocturnal cough & Weight gain (>2 kg/week) \\
Bendopnoea & Peripheral oedema (ankle, sacrum) \\
& Pulmonary crackles \\
\hline
\end{tabular}

Source: Atherton J, Sindone A, De Pasquale C, et al. National Heart Foundation of Australia and Cardiac Society of Australia and New Zealand Guidelines for the prevention, detection and management of heart failure in Australia 2018

drugs; BNP/ NT-ProBNP above HF cut-offs; recorded ejection fraction $(\mathrm{EF})<40 \%, \mathrm{EF} \geq 40-<50 \%$ and typical symptoms and signs recorded in the notes; and EF $\geq 40-<50 \%$ \& use of a loop diuretic The criteria for 'probable HF' were recorded EF $\geq 40-<50 \%$, or typical symptoms and signs of $\mathrm{HF}$ recorded in the notes \& any of the following: BNP/ NT-ProBNP in the inconclusive ranges, use of a loop diuretic, or documented EF > 50\%.

In Australia, the Federal Government mandates through the Pharmaceutical Benefits Scheme (PBS) that the HF-specific drugs used in our search and analysis are for to be prescribed for the management of heart failure only. General practitioners are unlikely to stray from these restrictions - education must be in line with the PBS listing and Medicare can perform audits on GPs' practices. Also, as HF is a clinical diagnosis that can also be inferred from response to treatment, it would be highly likely that patients with the constellation of symptoms described plus prescriptions for diuretic medication/s would have heart failure, even if no specific diagnosis has been entered or other more specific HF medications not initiated.

Data analysis was conducted using SAS for windows (version 9.4). For laboratory and other data, the most recent measurement for each patient of each parameter was selected for analysis. If any of the selected drugs were taken at any time by a patient during the whole period under study, then that patient was identified as having been prescribed that drug. Medications prescribed following the diagnosis of HF was also reviewed. Referrals (to a cardiologist, endocrinology or renal

Table 6 Pathology tests and indicative cut-offs for definite heart failure

\begin{tabular}{lll}
\hline & Test name & \\
\hline & BNP & NT-ProBNP \\
Heart failure rule-out & $<100 \mathrm{pg} / \mathrm{mL}$ & $<300 \mathrm{pg} / \mathrm{mL}$ \\
Heart failure rule-in & $>400 \mathrm{pg} / \mathrm{mL}$ & $>450 \mathrm{pg} / \mathrm{mL}$ age $<50 \mathrm{yrs}$ \\
& & $>900 \mathrm{pg} / \mathrm{mL}$ age $50-75 \mathrm{yrs}$ \\
& & $>1800 \mathrm{pg} / \mathrm{mL}$ age $>75 \mathrm{yrs}$ \\
\hline
\end{tabular}

Source: Atherton J, Sindone A, De Pasquale C, et al. National Heart Foundation of Australia and Cardiac Society of Australia and New Zealand Guidelines for the prevention, detection and management of heart failure in Australia 2018 
Table 7 Pathology cut-offs suggestive but inconclusive of heart failure (probable HF)

\begin{tabular}{lll}
\hline & Test name & NT-ProBNP \\
\hline Heart failure rule-out & BNP & $<300 \mathrm{pg} / \mathrm{mL}$ \\
Heart failure rule-in(probable HF) & $<100 \mathrm{pg} / \mathrm{mL}$ & $300-450 \mathrm{pg} / \mathrm{mL}$ age $<50 \mathrm{yrs}$ \\
& $>=100 \mathrm{pg} / \mathrm{mL}$ & $300-900 \mathrm{pg} / \mathrm{mL}$ age $50-75 \mathrm{yrs}$ \\
& & $300-1800 \mathrm{pg} / \mathrm{mL} \mathrm{age}>75 \mathrm{yrs}$ \\
\hline
\end{tabular}

Source: SHAPE Project Collaborators Expert Opinion, September 2018

physician) were recorded for a patient only if the referral occurred around the time of diagnosis of HF, or later. That period started one month prior to HF diagnosis and then onwards. This presumed that the referral to the specialist was the time when the GP was suspecting HF and was seeking specialist involvement. We also assessed referrals starting from seven months prior to diagnosis, which allowed for patients to have been seen by a specialist, provided with six months of prescriptions and so only needed a GP consultation after this period. In this case, the diagnosis may only appear in the GP records up to a maximum of seven months after the specialist visit.

The point prevalence and annual incidence of HF were calculated, along with their 95\% confidence intervals. From the age- and gender-specific rates of HF, and estimates of the Australian population in these subgroups, prevalence and incidence were age-standardised to the 2017 Australian population overall, and by gender.

The calculation of prevalence and incidence of HF involved only 'active' patients; that is, those patients with at least three visits per two-year period [11]. This approach avoided the under-estimation of prevalence and incidence that would have otherwise arisen from overinflation of the denominator data by one-off or infrequent GP visits. Such visits would be more common in bulk billing centres. Furthermore, among people who are not regular patients of the centres, medical records may not contain sufficient information on which to assess the presence of a HF diagnosis. In secondary analyses, denominators were estimated from the total number of patients seen at the participating GP clinics during each calendar year for the period under study.

Table 8 Criteria for stratification and number of patients by group

\begin{tabular}{|c|c|c|c|}
\hline \multirow[b]{2}{*}{ Group } & \multirow[b]{2}{*}{ Criteria } & \multicolumn{2}{|c|}{$\begin{array}{l}\text { Number of } \\
\text { patients }\end{array}$} \\
\hline & & All & $\begin{array}{l}\text { Active } \\
\text { only }\end{array}$ \\
\hline \multirow[t]{8}{*}{1 Patients who definitively had HF: } & - HF diagnosis recorded in the diagnosis/condition section, or & 3193 & 3026 \\
\hline & - HF diagnosis recorded or as free text in the notes, or & 8744 & 8103 \\
\hline & - Having had an HF-specific medication, or & 4773 & 4132 \\
\hline & - EF reduced (from free text in the notes), or & 144 & 137 \\
\hline & - BNP/ NT-ProBNP above HF cut-offs, or & 50 & 45 \\
\hline & - Recorded ejection fraction (EF) $<40 \%$, or & 12 & 11 \\
\hline & - EF $\geq 40-<50 \%$ and typical symptoms and signs recorded in the notes, or & 10 & 10 \\
\hline & - EF $\geq 40-<50 \%$ \& use of a loop diuretic & 4 & 4 \\
\hline \multirow{5}{*}{$\begin{array}{l}2 \text { Patients who had a probable } \\
\text { diagnosis of HF: }\end{array}$} & - $\mathrm{EF} \geq 40-<50 \%$, or & 19 & 19 \\
\hline & - Typical symptoms and signs recorded in the notes AND any of the following: & & \\
\hline & - BNP/ NT-ProBNP in the inconclusive ranges & 38 & 38 \\
\hline & - Use of a loop diuretic & 4754 & 4635 \\
\hline & - Documented EF > 50\% & 62 & 60 \\
\hline \multirow[t]{4}{*}{3 Patients where HF was possible: } & $\begin{array}{l}\text { - Two or more of the less typical symptoms and signs recorded either in the diagnosis/ } \\
\text { condition section or in the notes, or }\end{array}$ & 109 & 107 \\
\hline & $\begin{array}{l}\text { - Typical symptoms and signs recorded either in the diagnosis/condition section or in the } \\
\text { notes (only), or }\end{array}$ & $\begin{array}{l}36 \\
224\end{array}$ & 33,278 \\
\hline & - $\mathrm{EF}>50 \%$ or $\mathrm{EF}$ found in notes, but no percentage recorded, or & 100 & 100 \\
\hline & - BNP/ NT-ProBNP in the inconclusive ranges & 84 & 71 \\
\hline
\end{tabular}




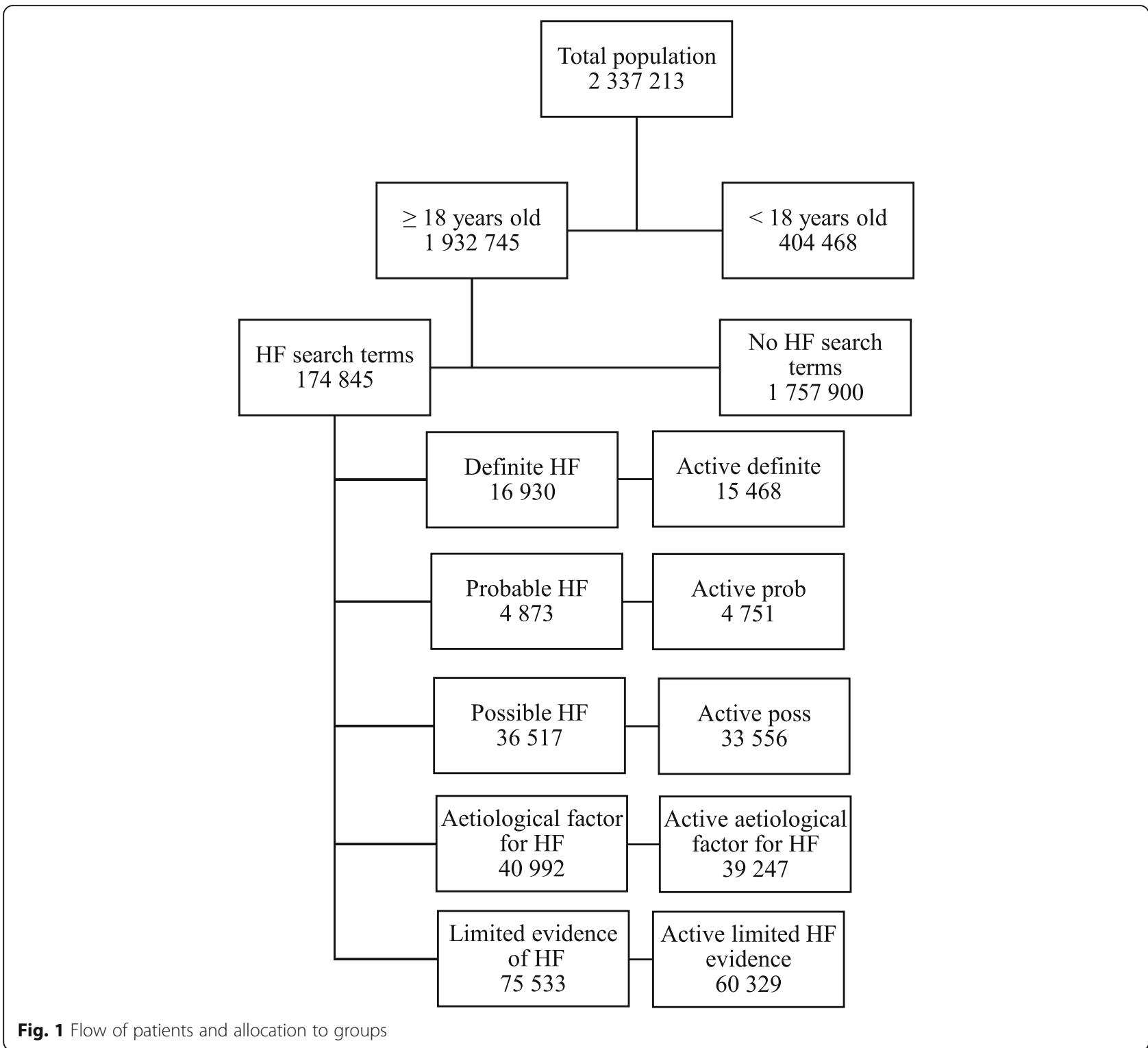

Table 9 Frequency of symptoms and signs of heart failure recorded in patients who had limited evidence of HF and were excluded from analysis

\begin{tabular}{ll}
\hline Symptoms or sign & Frequency recorded \\
\hline PND & $26.1 \%$ \\
Ankle oedema & $23.1 \%$ \\
Weight gain & $19.7 \%$ \\
Rales & $8.7 \%$ \\
Ankle swelling & $6.0 \%$ \\
Crepitations & $4.4 \%$ \\
Leg swelling & $4.0 \%$ \\
Pitting oedema & $1.7 \%$ \\
Leg oedema & $1.0 \%$ \\
Peripheral oedema & $1.0 \%$ \\
\hline
\end{tabular}

Overall prevalence within gender and age groups was calculated, along with the proportion of cases within each of the gender and age-groups.

The numerator for the prevalence of HF was obtained by tabulating the numbers of HF cases by age group and gender over the five-year period under study. Annual incidence of HF was reported similarly to prevalence, except that only new cases were included, based on the date of first diagnosis of HF. In an attempt to remove from the file the cases with pre-existing HF, we identified cases where the diagnosis of definite and probable HF was made or was present during the first year of data collection and removed these from the file. This meant that cases which remained in the incidence calculation had no mention of HF during the first year of the data collection. 
Table 10 Top 10 diagnosis terms recorded in the definite HF population

\begin{tabular}{|c|c|c|c|}
\hline & Diagnostic terms recorded in the notes & Number & Percent \\
\hline \multicolumn{4}{|c|}{ All patients } \\
\hline 1 & Congestive heart failure & 4663 & $27.5 \%$ \\
\hline 2 & Heart failure & 2457 & $14.5 \%$ \\
\hline 3 & Cardiac failure & 723 & $4.3 \%$ \\
\hline 4 & Heart failure \& Congestive heart failure & 672 & $4.0 \%$ \\
\hline 5 & Diastolic dysfunction & 370 & $2.2 \%$ \\
\hline 6 & Pulmonary oedema & 364 & $2.2 \%$ \\
\hline 7 & Cardiomyopathy & 217 & $1.3 \%$ \\
\hline 8 & Cardiac failure \& Congestive heart failure & 214 & $1.3 \%$ \\
\hline 9 & Ventricular failure & 194 & $1.1 \%$ \\
\hline 10 & Heart failure \& Cardiac failure & 169 & $1.0 \%$ \\
\hline \multicolumn{4}{|c|}{ Active patients } \\
\hline 1 & Congestive heart failure & 4393 & $28.4 \%$ \\
\hline 2 & Heart failure & 2177 & $14.1 \%$ \\
\hline 3 & Cardiac failure & 674 & $4.4 \%$ \\
\hline 4 & Heart failure \& Congestive heart failure & 639 & $4.1 \%$ \\
\hline 5 & Diastolic dysfunction & 356 & $2.3 \%$ \\
\hline 6 & Pulmonary oedema & 331 & $2.1 \%$ \\
\hline 7 & Cardiomyopathy & 208 & $1.3 \%$ \\
\hline 8 & Cardiac failure \& Congestive heart failure & 208 & $1.3 \%$ \\
\hline 9 & Ventricular failure & 181 & $1.2 \%$ \\
\hline 10 & Heart failure \& Cardiac failure & 160 & $1.0 \%$ \\
\hline
\end{tabular}

\section{Results}

The practices provided care to 2.3 million individual patients over the five-year period, of which 1.93 million were aged 18 years and above. Among these, 1.12 million were 'active' patients [11]. Based on the presence of one or more of the HF search terms (Table 2 to Table 7), full clinical data on 174,845 patients were extracted for further analyses. Of these patients, and based on the hierarchy of selection criteria (Table 8), 16,930 were classified as having 'definite HF', 4873 as having 'probable HF' and 36,517 as having 'possible HF' (Fig. 1). A further 40,992 were identified as having an aetiological condition associated with HF. The remaining 75,533 were initially identified for analysis on the basis of signs or symptoms of HF recorded as free text in the notes only. As these patients had limited evidence of HF, they were excluded from further analyses. The most frequent signs or symptoms from this group are displayed in Table 9, with paroxysmal nocturnal dyspnoea (PND), ankle oedema and weight gain being the most common.

For the active patients, 15,468 were classified as having 'definite HF', 4751 as having 'probable HF' and 33,
556 as having 'possible HF' (Fig. 1). A further 39,247 were identified as having an aetiological condition associated with HF.

It was not possible to identify the amount nor rate of weight gain to satisfy the ' $>2 \mathrm{~kg} /$ week' criterion and some other signs and symptoms, such as peripheral oedema and crepitations, were only included in the classification of possible HF if more than one of these signs or symptoms had been recorded.

The flow of patients in the search and evaluation process is shown in Fig. 1.

In the primary analysis of active patients, a formal HF diagnosis $(3026,19.5 \%)$, HF terms recorded as free text in the notes $(8103,52.4 \%)$ and HF-specific medication prescriptions $(4132,26.7 \%)$ were the most common methods to identify 'definite' HF patients (Table 8). Typical signs and symptoms of HF in combination with a diuretic prescription $(4635,97.6 \%)$ was the most common method to identify 'probable HF' patients. The vast majority of 'possible' HF patients $(33,278,99.2 \%)$ were identified by the presence of 2 or more of the typical signs or symptoms of HF (Table 8).

The most commonly recorded diagnostic terms for HF in the active population were 'congestive heart failure' (4393, 21.7\%), 'heart failure' (2177, 10.8\%) and 'cardiac failure' $(674,4.7 \%)$. Other frequently used terms included 'diastolic dysfunction', 'pulmonary oedema' and 'cardiomyopathy' (Table 10).

Terms such as 'HFrEF' and 'HFpEF', which have been in use for a few years, were not commonly noted. We found 19 records of these terms (one record of HFrEF, and 18 records of $\mathrm{HFpEF}$ ).

The most commonly prescribed HF-specific medications to the active 'definite' and 'probable' HF population were the beta blockers bisoprolol (3783, 18.7\%), carvedilol (957, 4.7\%) and nebivolol (736, 3.6\%), Table 11.

Signs and symptoms of HF were extracted from the free text of the consultation notes. Dyspnoea was by far the commonest recorded symptom in the active population (9401, 46.5\%), followed by the combination of dyspnoea \& paroxysmal nocturnal dyspnoea (638, $3.2 \%)$, paroxysmal nocturnal dyspnoea (550, 2.7\%) and the combination of dyspnoea \& orthopnoea (535, $2.6 \%)$, Table 12. The commonest recorded sign was elevated jugular venous pressure (JVP) in combination with dyspnoea $(117,0.6 \%)$, followed by a displaced apex beat $(100,0.5 \%)$ and elevated JVP alone (52, $0.3 \%)$, Table 12 .

Among the active population, the crude prevalence of definite or probable HF was $1.815 \%$ (95\% CIs 1.79$1.84 \%)$; and the age-standardised prevalence was 2.199\% (95\% CIs: $2.168-2.23 \%$ ). The crude incidence of definite or probable HF was $0.291 \%$ per year $(95 \%$ CIs 0.286-0.296\%), and the age-standardised incidence 
Table 11 Top 10 HF-specific medications prescribed in the definite HF population

\begin{tabular}{|c|c|c|c|}
\hline & HF-specific medications recorded in the notes & Number & Percent \\
\hline \multicolumn{4}{|c|}{ All patients } \\
\hline 1 & Bisoprolol & 4175 & $24.7 \%$ \\
\hline 2 & Carvedilol & 1093 & $6.5 \%$ \\
\hline 3 & Nebivolol & 793 & $4.7 \%$ \\
\hline 4 & Metoprolol succinate & 646 & $3.8 \%$ \\
\hline 5 & Ivabradine & 171 & $1.0 \%$ \\
\hline 6 & Bisoprolol \& Sacubitril & 113 & $0.7 \%$ \\
\hline 7 & Bisoprolol \& Carvedilol & 110 & $0.6 \%$ \\
\hline 8 & Eplerenone \& Bisoprolol & 110 & $0.6 \%$ \\
\hline 9 & Eplerenone & 77 & $0.5 \%$ \\
\hline 10 & Bisoprolol \& Nebivolol & 59 & $0.3 \%$ \\
\hline \multicolumn{4}{|c|}{ Active patients } \\
\hline 1 & Bisoprolol & 3783 & $24.5 \%$ \\
\hline 2 & Carvedilol & 957 & $6.2 \%$ \\
\hline 3 & Nebivolol & 736 & $4.8 \%$ \\
\hline 4 & Metoprolol succinate & 554 & $3.6 \%$ \\
\hline 5 & Ivabradine & 153 & $1.0 \%$ \\
\hline 6 & Bisoprolol \& Sacubitril & 111 & $0.7 \%$ \\
\hline 7 & Bisoprolol \& Carvedilol & 107 & $0.7 \%$ \\
\hline 8 & Eplerenone \& Bisoprolol & 101 & $0.7 \%$ \\
\hline 9 & Eplerenone & 72 & $0.5 \%$ \\
\hline 10 & Bisoprolol \& Nebivolol & 56 & $0.4 \%$ \\
\hline
\end{tabular}

was $0.348 \%$ per year (95\% CIs: $0.342-0.354 \%)$. The estimates of prevalence and incidence suggest that almost 420,000 people were living with HF in Australia in 2017, and over 66,000 new cases of HF occurred that year.

\section{Discussion}

Although medical record systems in the primary care setting can be well-structured, provider compliance with populating the records in accordance with the systems intended structure is variable and often incomplete [13]. We found that over $80 \%$ of patients identified as definite HF did not have a HF diagnosis recorded in the diagnosis section of their medical records, although over half (51.6\%) of the remainder had a diagnostic term recorded as free text in the consultation notes. Addressing this underuse of diagnostic codes is one of the goals of the Federal Health Department's new Practice Incentives Program (PIP) Quality Improvement (QI) incentive [14]. In this program, which commenced in August 2019, practices are rewarded for participating in continuous quality improvement activities in partnership with their local Primary Health Network (PHN). The areas chosen for improvement are to be informed by GPs' clinical information system data, from data collected by the PHN against specified Improvement Measures [14].

Despite a paucity of information on HF, previous studies of the HF epidemiology in Australia have shown consistent findings. In a recent article, investigators applied international data to Australian Bureau of Statistics population figures to estimate the prevalence $(2.1 \%)$ and annual incidence $(0.27 \%)$ of HF [1]. These figures were obtained by extrapolating from numbers of hospitalised cases, so that it may not be surprising that the figures presented in this paper were marginally higher than our own estimates. In the USA, prevalence of HF in the United States was reported to be $2.42 \%$ in 2012 [15]. A limited number of studies from Asian countries report prevalence estimates in the range 1.26-6.7\% [16]. Again, results in these studies were derived from demographic extrapolations of international data, often of hospitalised or post-hospitalised patients. In contrast, our estimates have been determined from medical records obtained from a general practice database that do not rely on hospitalisation as a marker for diagnosis. Accordingly, the patients in our dataset appear to be less co-morbid and on fewer evidence-based medications [17]. 
Table 12 Top 10 signs and symptoms of HF recorded in the definite and probable HF population

\begin{tabular}{|c|c|c|c|}
\hline & Sign/symptom combinations recorded in the notes & Number & Percent \\
\hline \multicolumn{4}{|c|}{ All patients } \\
\hline 1 & Dyspnoea & 9699 & $44.5 \%$ \\
\hline 2 & Dyspnoea \& PND & 646 & $3.0 \%$ \\
\hline 3 & PND & 561 & $2.6 \%$ \\
\hline 4 & Dyspnoea \& Orthopnoea & 549 & $2.5 \%$ \\
\hline 5 & Dyspnoea \& displaced apex beat & 231 & $1.1 \%$ \\
\hline 6 & Orthopnoea & 149 & $0.7 \%$ \\
\hline 7 & Dyspnoea, Orthopnoea \& PND & 130 & $0.6 \%$ \\
\hline 8 & Dyspnoea \& elevated JVP & 123 & $0.6 \%$ \\
\hline 9 & Displaced apex beat & 104 & $0.5 \%$ \\
\hline 10 & Elevated JVP & 58 & $0.3 \%$ \\
\hline \multicolumn{4}{|c|}{ Active patients } \\
\hline 1 & Dyspnoea & 9401 & $46.5 \%$ \\
\hline 2 & Dyspnoea \& PND & 638 & $3.2 \%$ \\
\hline 3 & PND & 550 & $2.7 \%$ \\
\hline 4 & Dyspnoea \& Orthopnoea & 535 & $2.6 \%$ \\
\hline 5 & Dyspnoea \& displaced apex beat & 225 & $1.1 \%$ \\
\hline 6 & Orthopnoea & 138 & $0.7 \%$ \\
\hline 7 & Dyspnoea, Orthopnoea \& PND & 124 & $0.6 \%$ \\
\hline 8 & Dyspnoea \& elevated JVP & 117 & $0.6 \%$ \\
\hline 9 & Displaced apex beat & 100 & $0.5 \%$ \\
\hline 10 & Elevated JVP & 52 & $0.3 \%$ \\
\hline
\end{tabular}

\section{Limitations}

As many of the clinics had transitioned from another practice software to Medical Director software over the course of the study period, it is possible that some diagnostic terms were lost in the transition process and not entered into the correct field in the Medical Director software.

Some data in the records are not available for electronic assessment as they are contained in scanned attachments in the systems (e.g. discharge summaries, echocardiogram reports) which may have reduced our ability to identify the presence and severity of heart failure and outcomes (eg rehospitalisation, death). As the point of diagnosis, treatment initiation and performance of key investigations may occur in the hospital setting, some patients may have been reclassified if the full hospital data had been available.

The use of programming methods to search free text for specific keywords is an inexact science. However, a number of records were reviewed manually to refine the search criteria and confirm that commonly appearing misspellings of words were correctly identified. It was not feasible to review a large number of patient notes (there were over 8 million records in total), but we believe that misclassification errors would have occurred infrequently so that the final results should be a good representation of the epidemiology in the Australian community setting.

It is known that approximately $13 \%$ of the population do not see their GP annually [18], and the likelihood is that very few of this group would have HF. Therefore, our figures for prevalence and incidence for the whole population may be overestimated, as they are based only on those patients who visit their GP actively.

Finally, in estimating HF incidence, prevalent cases were removed and those that remained for analysis had no mention of HF during the first year. This assumed that participating practices did not inherit new patients with existing HF during the subsequent years.

\section{Conclusions}

This novel approach to undertaking retrospective research of primary care data successfully analysed a combination of coded and uncoded data from the electronic medical records of patients routinely managed in the GP setting.

This has allowed us to produce the first definitive study of the epidemiology of HF in the general Australian community, quantifying the epidemiological characteristics of this population and providing valuable insight 
into the landscape of HF in Australian primary care, the SHAPE study.

Further analysis will inform on the current care of people with $\mathrm{HF}$ and provide guidance of how to improve their management.

A major issue facing such projects in the future is the issue of coding diseases. Our study found that the majority of patients with HF were not clinically coded for HF. Attention needs to be focused on supporting primary care to improve the entry of data into electronic medical records to enable better use and interpretation of these data.

\section{Supplementary information}

Supplementary information accompanies this paper at https://doi.org/10. 1186/s12889-020-08781-8.

Additional file 1. Appendix - Free text search terms.

\section{Abbreviations \\ ATC: Anatomical therapeutic chemical; BNP: Brain natriuretic peptide; DoH: Department of Health; EC: Ethic Committee; EF: Ejection fraction; EMR: Electronic medical record; GP: General practitioner; GPMP: GP Management plan; HF: Heart failure; HFrEF: Reduced ejection fraction heart failure (EF < 50\%); HFpEF: Preserved ejection fraction heart failure (EF $\geq 50 \%)$; HFuEF: Unquantified ejection fraction heart failure; JVP: Jugular venous pressure; LRTI: Lower respiratory tract infection; NT-proBNP: N terminal pro- brain natriuretic peptide; PHN: Primary Health Network; PND: Paroxysmal nocturnal dyspnoea; PIP: Practice Incentives Program; QI: Quality Improvement; URTI: Upper respiratory tract infection}

\section{Acknowledgements}

The authors would like to acknowledge the support of Patricia Berry of Novartis Australia Pty Ltd., who provided efficient project management for the study.

We are grateful to Healius Ltd. for their active involvement in the study, including the data identification and extraction processes.

\section{Authors' contributions}

$A S, R A, P P, D L, D H, A M N$ and RP contributed to data acquisition, data analysis, and reporting of the findings. $\mathrm{DL}, \mathrm{AMN}, \mathrm{RP}, \mathrm{AS}, \mathrm{DH}, \mathrm{RA}, \mathrm{PP}$ and $\mathrm{KL}$ were involved in drafting and critical revision of the manuscript. All authors read and approved the final version of the manuscript.

\section{Funding}

This study was sponsored by Novartis Pharmaceuticals Pty Limited, Australia. AusTrials was commissioned by Novartis Pharmaceuticals Australia Pty Ltd. to conduct the SHAPE study analyses. AMN and RWP are both employees of AusTrials.

Dr. Kevin Lim of Novartis Pharmaceuticals Pty Limited was involved in the study concept and design with Professors Danny Liew, Ralph Audehm, Andrew Sindone and Dr. Deepak Haikerwal.

Novartis Pharmaceuticals Pty Limited and its employees had no role in the data collection, development of the analysis plan, data analyses or interpretation of the data.

Dr. Kevin Lim and Ms. Patricia Berry of Novartis provided project management support.

Dr. Kevin Lim was involved in the manuscript writing.

\section{Availability of data and materials}

Data, which are derived from de-identified electronic medical records, are not publicly available and will not be made available to the general public. The data were provided by the participating medical centres belonging to an Australian health care company (Healius Ltd) which de-identified the data, removing all potentially identifiable data from the records, then provided to the researchers for analysis. Access to these data was granted by Healius following independent ethics approval of the study and institutional governance approval.

\section{Ethics approval and consent to participate}

The study was approved by the Bellberry Human Research Ethics Committee (Application No: 2018-09-746). The Healius Clinical Council provided governance approval for the study.

\section{Consent for publication}

Not applicable.

\section{Competing interests}

AS has received honoraria, speaker fees, consultancy fees, is a member of advisory boards or has appeared on expert panels for: Alphapharm, Aspen, AstraZeneca (AZ), Bayer, Biotronik, Boehringer Ingelheim, Bristol Myers Squibb, Janssen Cilag, Menarini, Merck Sharp and Dohme (MSD), Mylan, Novartis, Otsuka, Pfizer, Sanofi, Servier, Vifor. DH has given talks for AZ, Bayer, BMS, Novartis and Pfizer. PP has sat on advisory boards and/or spoken at, facilitated or chaired at meetings for and/or received travel and accommodation costs from AbbVie, Amgen, AZ, Bayer, Bl, BMS, CSL, Eli Lilly, GSK, Janssen, Menarini, MSD, Novartis, Novo Nordisk, Pfizer, Sanofi \& Seqirus. RA served as a member of several Advisory Boards and conducted paid presentations for AZ, Novartis and Sanofi in the past 2 years, and Abbott, BMS, Eli Lilly, Novo Nordisk, Servier and Takeda prior to this. DL has received honoraria from: Amgen, AZ, Bayer, BI, BMS, Novartis, Pfizer, Sanofi and Shire. AusTrials was commissioned by Novartis Pharmaceuticals Australia Pty Ltd. to conduct the SHAPE study. AMN and RWP are both employees of AusTrials. KL is a full-time employee of Novartis Pharmaceuticals Australia Pty Ltd.

\section{Author details}

'AusTrials, Brisbane, Australia. ${ }^{2}$ School of Public Health and Preventive Medicine, Monash University, Melbourne, Australia. ${ }^{3}$ Dept of General Practice, University of Melbourne, Melbourne, Australia. ${ }^{4}$ Heartwest, Melbourne, Australia. ${ }^{5}$ Five Dock Family Medical Practice, Sydney, Australia. ${ }^{6}$ Novartis Pharmaceuticals Pty. Ltd, Sydney, Australia. ${ }^{7}$ Director Heart Failure Unit and Department of Cardiac Rehabilitation, Concord Hospital, Sydney, Australia.

Received: 19 November 2019 Accepted: 26 April 2020

Published online: 11 May 2020

\section{References}

1. Chan YK, Tuttle C, Ball J, et al. Current and projected burden of heart failure in the Australian adult population: a substantive but still ill-defined major health issue. BMC Health Serv Res. 2016;16:501.

2. Australian Bureau of Statistics, Population Projections, Australia, 2012 to 2101 (cat. no. 3222.0), 2013.

3. Scott I, Jackson C. Chronic heart failure management in Australia - time for general practice centred models of care: AFP; 2013;42(5):343-46.

4. British Medical Association 2018. International models of general practice. www.bma.org.uk/-/media/files/pdfs/collective\%20voice/policy\%20research/ nhs\%20structure\%20and\%20delivery/international-models-of-generalpractice.pdf?la=en. Accessed 10 Nov 2019.

5. Krum H, Best M, Tonkin A, et al. Prevalence of Heart Failure and Use of BetaBlockers in Australian General Practice. Heart Lung Circ. 2012;S89(21):S1S142.

6. Cleland JGF, Cohen-Solal A, Cosin AJ. Management of heart failure in primary care (the IMPROVEMENT of heart failure Programme): an international survey. Lancet. 2002;360(9346):1631-9.

7. McInnes DK, Saltman DC, Kidd MR. General practitioners' use of computers for prescribing and electronic health records: results from a national survey. Med J Aust. 2006;185(2):88-91.

8. The Royal Australian College of General Practitioners. General Practice: Health of the Nation. East Melbourne: RACGP; 2017.

9. Medical Director. www.medicaldirector.com/help/index.htm\#t=kba\%2 Fclinical\%2FAn_Overview_of_MedicalDirector_Clinical.htm. Accessed 1 Nov 2019.

10. Atherton J, Sindone A, De Pasquale C, et al. National Heart Foundation of Australia and Cardiac Society of Australia and new Zealand guidelines for the prevention, detection and management of heart failure in Australia 2018 
11. Royal Australian College of General Practitioners. Standards for general practices. 5th ed. East Melbourne: RACGP; 2017.

12. Pharmaceutical Benefits Scheme, 2019. www.pbs.gov.au/medicine/item/ 8733P. Accessed 28 Oct 2019.

13. Gordon J, Miller G, Britt H. Deeble Institute Issues Brief No. 18: Reality check - reliable national data from general practice electronic health records emphasises the need to urgently address the lack of reliable national primary health data, particularly data from general practice; 2016.

14. Department of Health, PIP QI Incentive guidance, 2019. https://www1. health.gov.au/internet/main/publishing.nsf/Content/PIP-QI_Incentive_ guidance. Accessed 11 Aug 2019

15. Heidenreich PA, Albert NM, Allen LA, Bluemke DA, Butler J, Fonarow GC, et al. Forecasting the impact of heart failure in the United States: a policy statement from the American Heart Association. Circ Heart Fail. 2013;6(3): 606-19.

16. Sakata Y, Shimokawa H. Epidemiology of heart failure in Asia. Circ J. 2013; 77(9):2209.

17. Sindone, Haikerwal, Audehm, et al. A retrospective cohort study of heart failure in the Australian primary care setting (SHAPE) - clinical characteristics of HF patients. Adelaide: CSANZ; 2019.

18. Australian Institute of Health and Welfare. Medicare-subsidised GP, allied health and specialist health care across local areas: 2013-14 to 2017-18. https://www.aihw.gov.au/reports/primary-health-care/medicare-subsidisedgp-allied-health-and-specialis/contents/introduction. Accessed 20 Feb 2020.

\section{Publisher's Note}

Springer Nature remains neutral with regard to jurisdictional claims in published maps and institutional affiliations.

Ready to submit your research? Choose BMC and benefit from:

- fast, convenient online submission

- thorough peer review by experienced researchers in your field

- rapid publication on acceptance

- support for research data, including large and complex data types

- gold Open Access which fosters wider collaboration and increased citations

- maximum visibility for your research: over $100 \mathrm{M}$ website views per year

At $\mathrm{BMC}$, research is always in progress.

Learn more biomedcentral.com/submissions 